\title{
A Pharmacist-Driven Intervention Designed to Improve Medication Accuracy in the Outpatient Kidney Transplant Setting
}

This article was published in the following Dove Press journal:

Drug, Healthcare and Patient Safety

\author{
Elizabeth A Cohen' \\ Danielle McKimmy ${ }^{2}$ \\ Anna Cerilli ${ }^{3}$ \\ Sanjay Kulkarni $\mathbb{D}^{4}$
}

'Yale New Haven Transplant Center, Yale New Haven Hospital, New Haven, CT, USA; ${ }^{2}$ Department of Pharmacy, University of Florida Health Shands Hospital, Gainsville, FL, USA; ${ }^{3}$ Heart and Vascular Center, Yale-New Haven Hospital, New Haven, CT, USA; ${ }^{4}$ Section of Organ Transplantation, Yale University School of Medicine, New Haven, CT, USA

Correspondence: Elizabeth A Cohen Yale New Haven Transplant Center, 333 Cedar Street, New Haven, CT 065II, USA

Tel +I 203-200-4578

Email Elizabeth.cohen@ynhh.org
Background: Medication errors are one of the leading causes of complications and readmissions in healthcare and stem directly from inadequate medication lists. In transplantation, medication discrepancies can lead to fluctuating levels of immunosuppression, resulting in rejection, infection, or drug toxicity.

Methods: We implemented a pharmacist-driven intervention designed to improve the accuracy of outpatient kidney transplant patients' medication lists in the electronic medical record (EMR). Baseline medication error rates (Phase 1) were collected, and the intervention was a dedicated pharmacist (Phase 2) who performed medication reconciliation with patients. The primary outcome was the percent of patients with inadequate medication reconciliation determined by any one error in medication reconciliation (Phase 1 vs Phase 2). Secondary outcomes included the number of medication errors, of all medications and high-risk medications, identified per patient sample using statistical process control phase analysis.

Results: Pharmacist-driven medication reconciliation significantly reduced medication list discrepancies from $95 \%$ to $28 \%(P<0.05)$. There were a total of 398 errors in the control group and 49 errors in the intervention group. In addition, there were 73 high-risk medication discrepancies in the control group and three in the intervention group. The total number of medication errors decreased post-intervention with a marked reduction in the variation of control limits (LCL, UCL: phase 1, -34.3, 113.9; phase 2, -7.1, 15.3) and average number of medication errors per sample (phase 1, 39.8; phase 2, 14.1). For high-risk medications, phase analysis demonstrated a marked reduction in control limit variation between phases (LCL, UCL: phase 1, $-10.4,25.0$; phase $2,-0.5,0.7$ ) and average number of medication errors per sample (phase 1, 7.3; phase 2,0.1).

Discussion: A dedicated pharmacist improved medication list accuracy over conventional practice that utilizes transplant nurses and physicians. Further studies into the costeffectiveness of this strategy should further justify this approach.

Keywords: kidney transplant, immunosuppression, medication reconciliation

\section{Introduction}

Medication reconciliation is defined by the Institute for Health Care Improvement as

the process of creating the most accurate list possible of all medications a patient is taking including drug name, dosage, frequency, and route and comparing that list against the physician's admission, transfer, and/or discharge orders, with the goal of providing correct medications. ${ }^{1}$ 
Inaccuracies in medication reconciliation are thought to cause up to $40 \%$ of medication errors, the leading cause of mistakes that cause patient harm, and has been an important target for quality improvement in the hospital and transitions of care settings. ${ }^{2}$ The incorporation of electronic medical records (EMRs) can help decrease these inaccuracies, but there are still a number of limitations. EMRs cannot always communicate between different health systems, and pharmacy records are often on different EMR platforms. Pharmacists are in an excellent position to help decrease errors on patient's medication lists, which has been shown in the inpatient setting. Reeder and Mutnick $^{3}$ demonstrated that pharmacistobtained medication histories on an inpatient internal medicine service were significantly more accurate than those obtained by physicians. This is especially important as patients have increasing web access to their EMR and often use this information to validate their medication list, especially on hospital discharge. If these have not been updated accurately, patients could take the wrong doses or even inappropriate medications. A review of published studies in the ambulatory care setting demonstrates that there are a small number of differing initiatives of pharmacist-driven reconciliation interventions, particularly in the primary care setting, but not in transplantation. ${ }^{12}$

In the kidney transplant setting, there is an especially high impact that may result from medication errors, which may directly lead to graft loss. ${ }^{4}$ It has been demonstrated that if patients are exposed to lower levels of tacrolimus, they are at higher risk of developing donor specific antibodies, ${ }^{5}$ whose development are associated with graft loss. ${ }^{6}$ Friedman et $\mathrm{al}^{7}$ reported on medication errors in an abdominal transplant population and found that one-third of errors were identified at the health-system level, with some errors leading to unnecessary invasive procedures and hospitalizations. Given that several medication changes in immunosuppression, comorbid condition management, and prophylaxis medications occur in the outpatient setting post-transplant, the maintenance of accurate medication lists has the potential to directly impact outcomes.

The purpose of this study was to evaluate a pharmacistdirected intervention designed to provide medication reconciliation and determine if this resulted in more accurate medication lists compared to conventional practices where medication management was performed by transplant nurses and physicians.

\section{Patients and Methods}

The Yale University Institutional Review Board approved this study through a waiver and informed consent was not required. All procedures were performed in accordance with relevant guidelines and procedures. Patient data was maintained with confidentiality on a HIPAA compliant server. To obtain baseline medication error rates, we used computerized block randomization of all completed kidney transplant clinic visits over a 2-month period, eliminating duplicate visits, hospitalized patients, and recipients of dual organ transplants. Ten blocks of 10 random patients $(\mathrm{n}=100)$ were provided to the pharmacy team. The pharmacist retrospectively performed a best possible medication history reviewing the EMR medication lists and comparing it to patient-reported medication lists via a telephone encounter within 2 weeks after the patient clinic visit. The patient-reported information was then confirmed with the patients' outpatient pharmacy. An error was defined as any discrepancy in the EMR in medication omission, addition, dose, duplication, frequency, formulation, route, substitution, or name when compared to the best possible medication history of patient-report and pharmacy records. In instances where there were unrecognized generic substitutions, these were not designated as errors as long as dosing and frequency were consistent. All discrepancies were documented and resolved in the EMR along with the data collection tool.

Figure 1 shows a schematic representation of the study. Control samples included standard of care kidney transplant medication management, which is performed by transplant nurses and physicians during an outpatient clinic visit. Following control group (Phase 1) error determination from the randomized patient samples, the pharmacy-directed intervention (Phase 2) was implemented and conducted prospectively. The intervention used a dedicated pharmacist who saw randomly selected patients at the beginning of regularly scheduled in-person clinic visits and performed the medication reconciliation with each patient, while updating the patient's EMR medication list. This differs from the standard practice of having nurses perform medication histories and physicians performing medication reconciliation. Given the fact that transplant teams need to cover several aspects of post-transplant care, our rationale was that a dedicated resource to assure medication reconciliation would translate into greater accuracy in patient medication lists. All recorded errors were shared with the primary care team and the patient's pharmacy was contacted to assure that prescriptions 


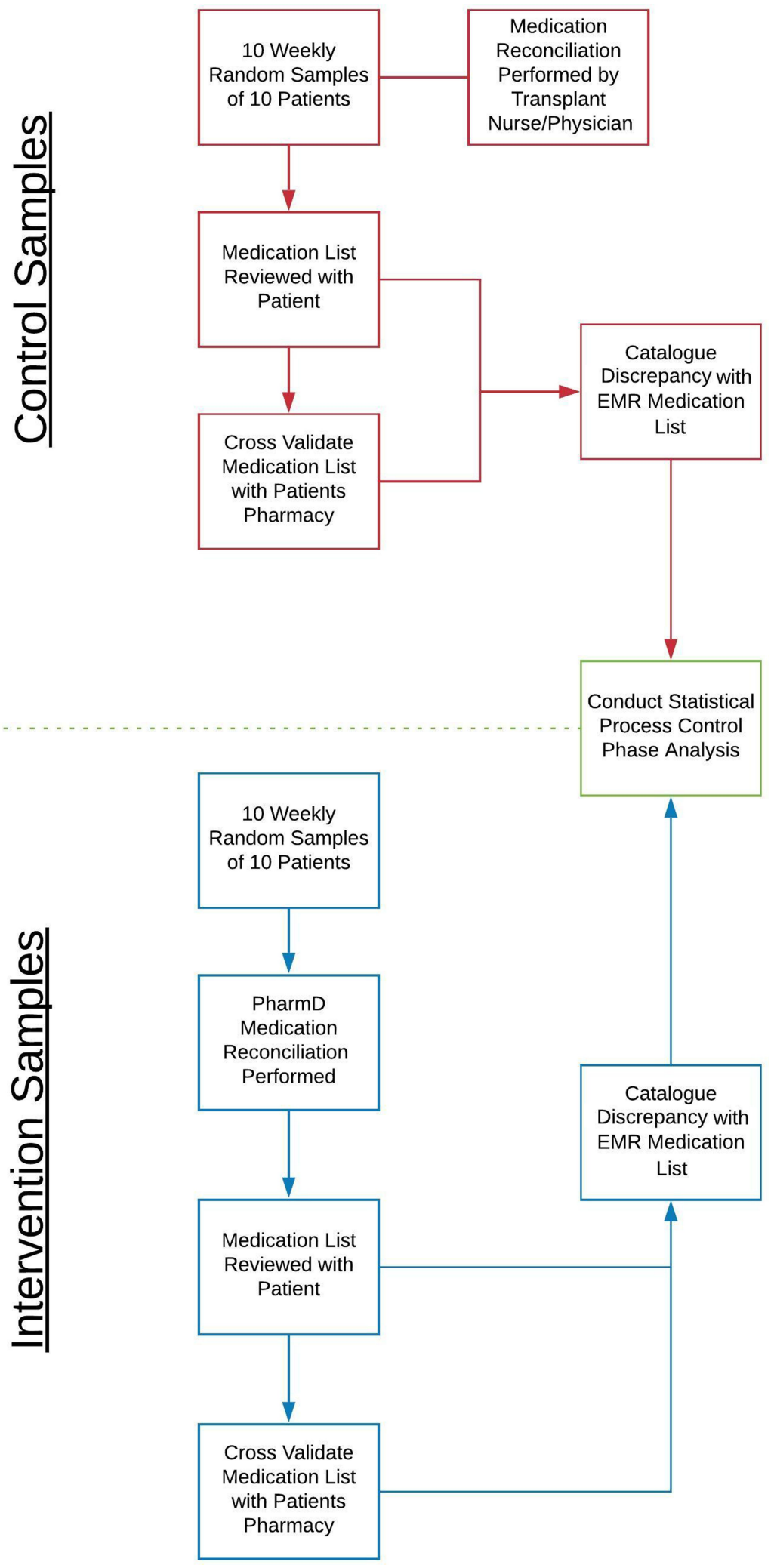

Figure I Control and intervention methods. 
Table I Patient Demographics

\begin{tabular}{|l|l|l|l|}
\hline & Phase I (n= 100) & Phase 2 (n= 100) & P-value \\
\hline Male (n, \%) & $69(69 \%)$ & $52(52 \%)$ & 0.021 \\
\hline Age at visit (average, range) & $58(25.7-82.9)$ & $54.4(20.8-76.2)$ & 0.049 \\
\hline $\begin{array}{l}\text { Ethnicity } \\
\text { White }\end{array}$ & $56(56 \%)$ & $47(47 \%)$ & 0.060 \\
Black & $31(31 \%)$ & $25(25 \%)$ & \\
Hispanic & $8(8 \%)$ & $23(23 \%)$ & $4(4 \%)$ \\
Asian & $5(5 \%)$ & $3.4(0-36)$ & 0.102 \\
\hline Time post-transplant (average [years], range) & $4.7(0.1-31.9)$ & $13.1(5-33)$ & 0.535 \\
\hline Number of medications (average, range) & $12.5(4-31)$ & $4.1(2-11)$ & 0.017 \\
\hline Number of high-risk medications (average, range) & $3.5(2-7)$ & & \\
\hline
\end{tabular}

accurately reflected the results of the medication reconciliation. Following the clinic visits, the same patients underwent audits via telephone encounters by the pharmacist within 2 weeks after their clinic visit of their medication lists to assess if any discrepancies were missed during the clinic visit. Errors were again determined if there was a mismatch in medication type, dosing, or frequency. High-risk medications were separately catalogued and defined as immunosuppressants, anticoagulants, antiarrhythmics, insulins, opioids, or benzodiazepines. All discrepancies were documented and resolved in the EMR.

Our primary outcome was the percent of patients with inadequate medication reconciliation determined by any one error in medication reconciliation (Phase 1 vs Phase 2). Secondary outcomes included the number of medication errors identified per patient sample and included an analysis of all medications and high-risk medications. Statistical process control phase analysis, which is an established methodology to assess quality improvement, was conducted to provide process-related assessment of our intervention. ${ }^{8}$ The number of medication errors per patient sample of 10 separate randomized samples were used to determine differences in errors between the control and intervention group. Phase analysis was performed using JMP Pro 13 (SAS Institute, Cary, NC). Upper control limits (UCL) and lower control limits (LCL) were conventionally set by the software to show three sigma ranges above and below the mean. Assessment of the effect of the intervention in the phase analysis was determined by the reduction in variability in errors detected, which was reflected by a difference between UCL and LCL's in both phases. Student's $t$-test was used as descriptive statistics for continuous data and chi-squared was used to determine statistical significance in the primary outcome.

\section{Results}

\section{Demographics}

Table 1 shows demographic information of Phase 1 and Phase 2 patients. Phase 2 had more patients who are female and patients who are Hispanic, with a shorter time from transplant $(P<0.05)$. Block randomization was independently performed to identify patients within Phase 1 and Phase 2, not for group assignment, therefore some differences in demographics are expected, but were not deemed clinically significant.

\section{Outcomes}

Table 2 shows the primary and secondary outcomes. Ninety-five percent of patients in the control group vs $28 \%$ of patients in the intervention group had at least one medication reconciliation error, which was statistically significant $(P<0.05)$. There were a total of 398 errors in the control group and 49 errors in the intervention group. In addition, there were 73 high-risk medication discrepancies in the control group and three in the intervention group.

\section{Statistical Process Control}

Phase analysis was performed from control charts that showed the number of medication discrepancies observed. Figure 2 shows the total number of medication errors decreased postintervention with a marked reduction in the variation of control limits (LCL, UCL: phase 1, -34.3, 113.9; phase 2, -7.1, 15.3) and average number of medication errors per sample (phase 1 39.8; phase 2, 14.1). For high-risk medications 
Table 2 Medication Discrepancy Outcomes

\begin{tabular}{|l|l|l|}
\hline & Phase I (n= 100) & Phase 2 (n= 100) \\
\hline Inadequate Medication Reconciliation, $n$ (\%) & $95(95 \%)$ & $28(28 \%)$ \\
\hline Total Discrepancies (n) & 398 & 49 \\
\hline Discrepancies (n) & & \\
Average per patient & 4 & 0.5 \\
Minimum per patient & 0 & 0 \\
Maximum per patient & 16 & 5 \\
\hline High-risk discrepancies (n) & & \\
Total & 73 & 3 \\
Average per patient & 0.75 & 0.03 \\
Minimum per patient & 0 & 0 \\
Maximum per patient & 4 & 1 \\
\hline
\end{tabular}

including immunosuppressants, anticoagulants, antiarrhythmics, insulins, opioids, or benzodiazepines, phase analysis is shown in Figure 3, which demonstrated a marked reduction in control limit variation between phases (LCL, UCL: phase 1, $-10.4,25.0$; phase $2,-0.5,0.7)$ and average number of medication errors per sample (phase 1, 7.3; phase 2, 0.1).

\section{Discussion}

The pharmacist role in the transplant team continues to evolve. It has been demonstrated by other transplant groups that the addition of clinical pharmacy services to a kidney transplant team improved inpatient medication management, medication reconciliation, discharge planning, patient education, and adherence. ${ }^{9,10}$ Our data adds an additional benefit of the transplant pharmacist, specifically in the outpatient setting to improve medication reconciliation over standard of care where this is performed by transplant nurses and physicians inclusive of a post-transplant clinic visit. Post-transplant management often involves several medication adjustments and changes, so an intervention designed to improve medication accuracy in this setting may have a significant impact on mitigating complications associated with medication errors.

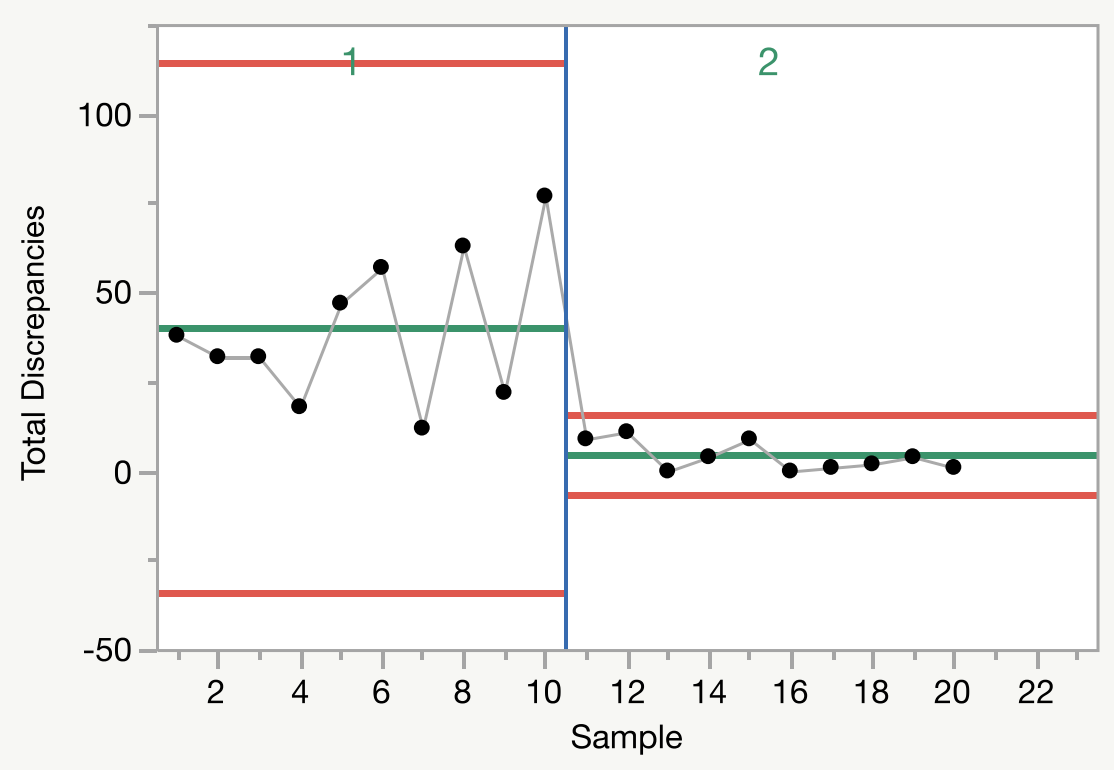

Figure 2 Total medication errors Phase I (control) vs Phase 2 (intervention). 


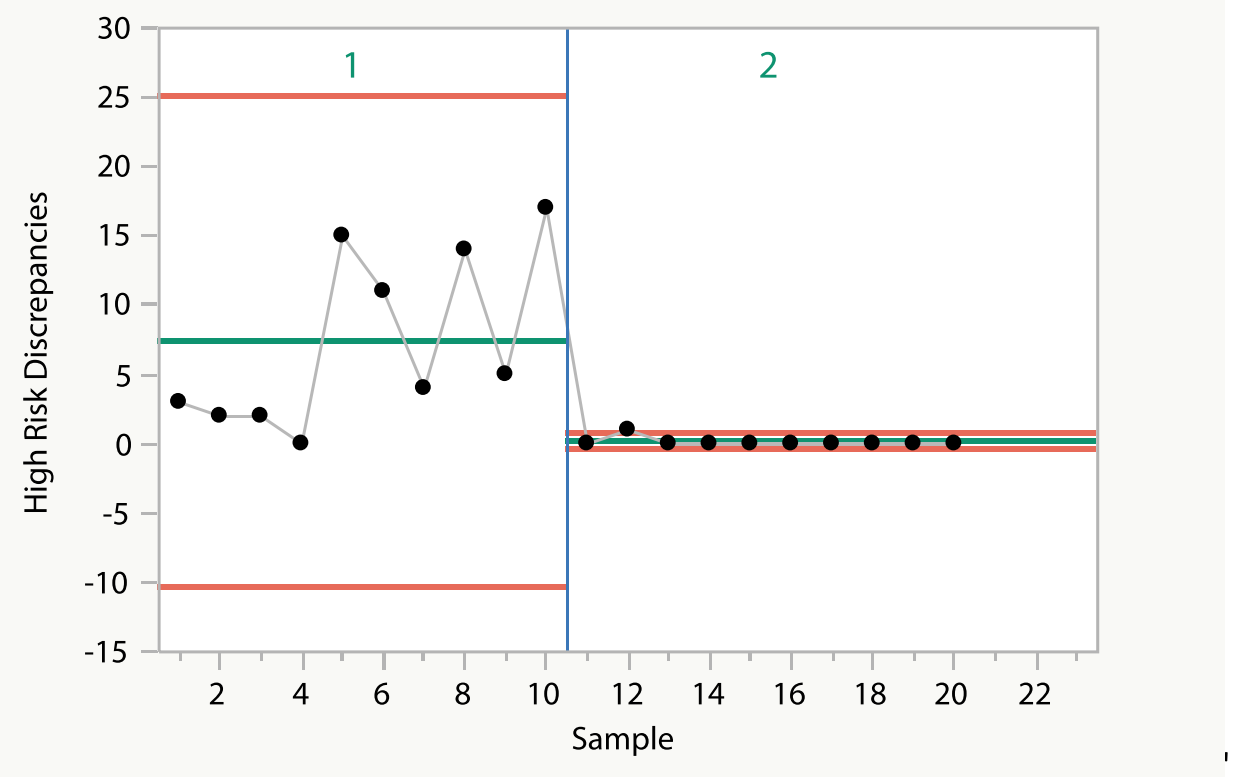

Figure 3 High-risk medication discrepancies Phase I (control) vs Phase 2 (intervention).

Our results demonstrated a decrease in medication discrepancies from $95 \%$ in Phase 1 to $28 \%$ in Phase 2 $(P<0.05)$. The discrepancies that still existed despite the pharmacist-directed intervention were primarily discrepancies in herbal medications, especially specific product and dose errors, as patients often did not know this information in clinic, but reported them when called at home. The high-risk medication discrepancies were deemed the most clinically relevant as these included immunosuppressives where higher or lower levels could have significant adverse events such as rejection, infection, or drug toxicity. Supporting our primary outcome findings, we performed process-related phase analysis using statistical process control, a methodology that has been advocated in the healthcare setting. ${ }^{8}$ Interpretation of the effect of the intervention is presented in Figure 2, where a reduction in variability between the UCL and LCL can be seen following implementation of our intervention.

We acknowledge the following study limitations. First, although Phase 2 was prospective, baseline error determination for Phase 1 was retrospective, resulting in the inability to robustly establish causation. Second, we did not determine which patients used their EMR remotely for medication list validation, which could have improved accuracy in this group. However, we note that the effect size was clinically relevant, particularly when determining accuracy of high-risk medications. Third, it is possible that transplant staff members, who were not blinded to the intervention, changed clinical practice knowing that medication lists were being reviewed and errors measured. This limitation exists and would be very difficult to control for. Lastly, statistical process control is methodology used to determine if errors in process fall significantly beyond expected trend lines. It does not allow for the adjustment of several factors, such as age, race, gender, and education level that could impact a healthcare intervention. However, we note that the differences in demographics noted were small and unlikely to impact outcomes.

We acknowledge that this intervention may or may not be cost neutral depending on the magnitude of cost reductions associated with rejection and a failing graft, which this study did not determine. However, reductions in medication discrepancies by $52 \%$ and accounting for costs associated with preventable adverse drug events translate into a net benefit of $\$ 206$ per patient, even considering the cost of the pharmacist. ${ }^{11}$ Although we did not evaluate adverse drug events in this evaluation, our reduction in medication discrepancies was higher than those previously published. ${ }^{9,10}$ There is likely a substantial cost saving with the implementation of the pharmacist-driven medication reconciliation on the healthcare system.

Our data demonstrates that pharmacists dedicated to performing medication reconciliation post-transplant significantly decreases the number of medication discrepancies, including immunosuppressives and other high-risk medications. Pharmacist intervention in medication 
reconciliation is ready to be implemented in other sites, but is limited by the availability of pharmacists in the ambulatory setting. Further studies are needed to assess the impact of these discrepancies on medication errors, clinical outcomes, and overall cost of care.

\section{Abbreviations}

EMR, electronic medical record; LCL, lower control limits; UCL, upper control limits.

\section{Acknowledgments}

The authors would like to thank the transplant quality improvement team for making this project possible.

\section{Disclosure}

The authors have nothing to disclose regarding the content of this manuscript.

\section{References}

1. Medication reconciliation to prevent adverse drug events. Available from: http://www.ihi.org/Topics/ADEsMedicationReconciliation/ Pages/default.aspx. Accessed November 6, 2020.

2. Abdulghani KH, Aseeri MA, Mahmoud A, Abulezz R. The impact of pharmacist-led medication reconciliation during admission at tertiary care hospital. Int J Clin Pharm. 2018;40(1):196-201. doi:10.1007/ s11096-017-0568-6

3. Reeder TA, Mutnick A. Pharmacist- versus physician-obtained medication histories. Am J Health Pharm. 2008;65(9):857-860. doi:10.2146/ajhp070292
4. Taber DJ, Pilch NA, Bratton CF, McGillicuddy JW, Chavin KD, Baliga PK. Medication errors and adverse drug events in kidney transplant recipients: incidence, risk factors, and clinical outcomes. Pharmacotherapy. 2012;32(12):1053-1060. doi:10.1002/phar.1145

5. Davis S, Gralla J, Klem P, et al. Lower tacrolimus exposure and time in therapeutic range increase the risk of de novo donor-specific antibodies in the first year of kidney transplantation. $A m$ $J$ Transplant. 2018;18(4):907-915. doi:10.1111/ajt.14504

6. Wiebe C, Gibson IW, Blydt-Hansen TD, et al. Rates and determinants of progression to graft failure in kidney allograft recipients with de novo donor-specific antibody. Am $J$ Transplant. 2015;15 (11):2921-2930. doi:10.1111/ajt.13347

7. Friedman AL, Geoghegan SR, Sowers NM, Kulkarni S, Formica RN Jr. Medication errors in the outpatient setting: classification and root cause analysis. Arch Surg. 2007;142(3):278-283; discussion 284. doi:10.1001/archsurg.142.3.278

8. Benneyan JC, Lloyd RC, Plsek PE. Statistical process control as a tool for research and healthcare improvement. Qual Saf Health Care. 2003;12(6):458-464. doi:10.1136/qhc.12.6.458

9. Alloway RR, Dupuis R, Gabardi S, et al. Evolution of the role of the transplant pharmacist on the multidisciplinary transplant team. Am $J \quad$ Transplant. 2011;11(8):1576-1583. doi:10.1111/j.16006143.2011.03601.x

10. Chisholm MA, Mulloy LL, Jagadeesan M, DiPiro JT. Impact of clinical pharmacy services on renal transplant patients' compliance with immunosuppressive medications. Clin Transplant. 2001;15 (5):330-336. doi:10.1034/j.1399-0012.2001.150505.x

11. Maldonado AQ, Weeks DL, Bitterman AN, et al. Changing transplant recipient education and inpatient transplant pharmacy practices: a single-center perspective. Am $J$ Health Pharm. 2013;70 (10):900-904. doi:10.2146/ajhp120254

12. McCarthy L, Su X, Crown N, et al. Medication reconciliation interventions in ambulatory care: a scoping review. Am J Health Pharm. 2016;73(22):1845-1857. doi:10.2146/ajhp150916
Drug, Healthcare and Patient Safety

\section{Publish your work in this journal}

Drug, Healthcare and Patient Safety is an international, peer-reviewed open-access journal exploring patient safety issues in the healthcare continuum from diagnostic and screening interventions through to treatment, drug therapy and surgery. The journal is characterized by the rapid reporting of reviews, original research, clinical, epidemiological and post-marketing surveillance studies, risk management, health literacy and educational programs across all areas of healthcare delivery. The manuscript management system is completely online and includes a very quick and fair peer-review system. Visit http://www.dovepress.com/testimonials.php to read real quotes from published authors. 